\title{
MALDI Mass Spectrometry Imaging Reveals Heterogeneous Distribution of Tenofovir and Tenofovir Diphosphate in Colorectal Tissue of Subjects Receiving a Tenofovir-Containing Enema
}

\author{
D Herana Kamal Seneviratne, (1) Craig W. Hendrix, Edward J. Fuchs, \\ and (1) Namandjé N. Bumpus \\ Department of Medicine, Division of Clinical Pharmacology, The Johns Hopkins University School of Medicine, Baltimore, \\ Maryland
}

Received May 3, 2018; accepted July 18, 2018

\begin{abstract}
Efforts to prevent human immunodeficiency virus (HIV) infection via pre-exposure prophylaxis (PrEP) include the development of anti-HIV drugs as microbicides for topical application to the mucosal sites of infection; however, although understanding the distribution profiles of these drugs in target mucosal tissues is of critical importance to guiding their optimization, data in this regard are largely lacking. With this in mind, we developed a matrixassisted laser desorption/ionization mass spectrometry imaging (MALDI MSI) approach to visualize tenofovir (TFV), an HIV nucleotide analog reverse-transcriptase inhibitor under investigation for use as a topical microbicide, and its active metabolite TFVdiphosphate (TFV-DP) in colorectal biopsies obtained from healthy volunteers who received TFV-containing enemas. Application of MALDI MSI resulted in sufficient spatial resolution to
\end{abstract}

visualize both TFV and TFV-DP and revealed heterogeneity in the distribution profiles of both analytes, including the presence of regions in which TFV and TFV-DP were undetectable, in colorectal tissue at two different time points and concentrations. Cellspecific staining for CD4 T and CD11c dendritic cells, which are important to the establishment of HIV infection, demonstrated that the TFV and TFV-DP distributions were independent of these cell types. MALDI MSI of endogenous lipids demonstrated that the heterogeneity observed for TFV and TFV-DP was not a function of tissue composition or processing. These data provide unique insight into the spatial distribution of TFV and TFV-DP in human colorectal tissue. In addition, this work establishes an approach that can be leveraged to directly detect and visualize these clinically important analytes more broadly in tissue.

\section{Introduction}

Tenofovir (TFV), as the prodrug TFV disoproxil fumarate (TDF), is currently used orally in combination with other antiretroviral drugs for human immunodeficiency virus (HIV) treatment and pre-exposure prophylaxis (PrEP) (Robbins et al., 1998; Miller et al., 2001; Kearney et al., 2004; De Clercq, 2007). TDF is converted first to a monoester and then to TFV upon the action of a carboxylesterase and a phosphodiesterase, respectively (Naesens et al., 1998; Kearney et al., 2004). Subsequently, TFV is phosphorylated first into TFV-monophosphate and then to TFV-diphosphate (TFV-DP), to become pharmacologically active (Robbins et al., 1998; Lade et al., 2015). Our laboratory has previously demonstrated that adenylate kinase 2 can mediate the first phosphorylation step in vaginal, colorectal, and peripheral blood mononuclear cells (Lade et al., 2015). Additionally, an enzyme denoted as creatine kinase muscle catalyzes the reaction that converts TFV-monophosphate into TFV-DP in

This research was funded by the National Institutes of Health [Grants U19AI11327, UM1AI068613, R01AI128781].

https://doi.org/10.1124/jpet.118.250357. colorectal tissue, whereas pyruvate kinase muscle and pyruvate kinase liver and red blood cells can facilitate the formation of TFV-DP in peripheral blood mononuclear cells and vaginal tissue (Lade et al., 2015).

Despite the effectiveness of oral daily TDF/emtricitabine PrEP, many at-risk individuals are interested in alternative dosing formulations owing to struggles with oral daily adherence, desire for single on-demand dosing, and desire to avoid systemic side effects associated with oral dosing, among other reasons (Bauermeister et al., 2013; Dolezal et al., 2015; Eaton et al., 2015; Mitchell et al., 2016; Carballo-Diéguez et al., 2017; Greene et al., 2017). Of note, the practice of rectal douching with enemas in association with receptive anal intercourse (RAI) is common among men who have sex with men (MSM). Therefore, high acceptability of rectal microbicides delivered via enema is expected among MSM. Thus, there is an urgent need to develop a safe, effective, and acceptable rectal microbicide for preventing rectally acquired HIV infection. In this regard, recognizing the importance of adherence in determining the effectiveness of PrEP, our group is contributing to efforts to develop an on-demand, behaviorally congruent

ABBREVIATIONS: CHCA, $\alpha$-cyano-4-hydroxycinnamic acid; HIV, human immunodeficiency virus; MALDI MSI, matrix-assisted laser desorption/ ionization mass spectrometry imaging; MSM, men who have sex with men; PrEP, pre-exposure prophylaxis; RAI, receptive anal intercourse; TDF, tenofovir disoproxil fumarate; TEC, tissue extinction coefficient; TFV, tenofovir; TFV-DP, tenofovir diphosphate. 
rectal TFV microbicide to prevent HIV acquisition via unprotected RAI.

TFV has been investigated for topical application to rectal and/or vaginal tissue for PrEP (Abdool Karim et al., 2010; Anton et al., 2012). Several PrEP clinical trials, such as CAPRISA 004, iPrEx, TDF2, Partners PrEP, VOICE, and FEM-PrEP, have been conducted using TFV gel or oral TDF (Baeten and Celum, 2013). Interestingly, the outcomes of above studies were widely different. With the above variability in study outcomes in mind, gaining a more complete understanding of the disposition of TFV in mucosal tissues that are putative sites of HIV infection is of importance. Specifically, an understanding of TFV and TFV-DP distribution in tissue is required to optimize the development of $\mathrm{TFV}$ as a topical approach to PrEP.

To date, characterization of TFV disposition in tissue has relied solely on data obtained using tissue homogenates or cells extracted from tissue biopsies. While of value for understanding overall drug concentrations in tissue, analyses performed using whole biopsy homogenates or extracted cells alone are unable to reveal differences in TFV and TFV-DP distribution across regions of tissue, including regions that may be devoid of drug. Since heterogeneity in drug distribution and/or regional variability in drug concentration could impact prophylactic activity, approaches that are able to monitor the presence of a drug in tissue in at least twodimensions are required. To address this, we developed a matrix-assisted laser desorption/ionization mass spectrometry imaging (MALDI MSI) method to visualize TFV and TFV-DP in tissue. MALDI MSI is a powerful technique to apply in this setting as it allows for simultaneous mapping of molecules that differ in mass without the use of compoundspecific labels (Greer et al., 2011; Angel and Caprioli, 2013). In the present study, we used colorectal biopsies obtained from healthy volunteers who received TFV-containing enemas, and the developed MALDI MSI method was applied to determine the localization of TFV and TFV-DP. Through this work, we demonstrated that the distribution of TFV and TFV-DP in colorectal tissue was heterogeneous, and as such TFV and TFV-DP are present at differential concentrations across tissue. These data provide a foundation for understanding the spatial distribution of TFV and TFV-DP in human tissue and importantly raise the possibility that the disposition of TFV and TFV-DP cannot be fully understood through the application of approaches that measure average concentrations in tissue alone.

\section{Materials and Methods}

Materials/Chemicals. TFV and TFV-DP were purchased from Toronto Research Chemicals, Inc., (North York, ON, Canada). MALDI matrices, $\alpha$-cyano-4-hydroxycinnamic acid (CHCA), 2, 5-dihydroxybenzoic acid, 3-hydroxypicolinic acid, and 9-aminoacridine were obtained from MilliporeSigma (St. Louis, MO). All solvents and other chemicals used were either reagent or high-performance liquid chromatography (HPLC) grade and purchased from Fisher Scientific (Hampton, NH), unless otherwise specified.

Biopsy Samples. DREAM-01 is an early-phase 1, multicenter, dose-escalation study in which three formulations of TFV were tested. All human subjects involved in this study were enrolled at the Johns Hopkins University and provided written informed consent prior to the study. The DREAM-01 study protocol was approved by the Johns Hopkins Medicine Institutional Review Board (JHMIRB) and conducted according to the ethical principles indicated in the Declaration of Helsinki. The study recruited HIV-negative, MSM healthy volunteers, age 18 or older, who had a history of engaging in RAI. Colorectal tissues samples were collected on the basis of a sparse sampling schedule assigned to each research participant. The first four participants at Johns Hopkins (J001-J004) were assigned to colorectal tissue collection at 3 and 24 hours following enema administration owing largely to sample accessibility. Pinch biopsies were collected via a flexible sigmoidoscope at the designated sampling times following enema dosing. All biopsies were collected at approximately $10-20 \mathrm{~cm}$ from the anal sphincter using large-cup biopsy forceps. Biopsies in this study were taken after two different enema doses were administrated at 1.76 (low-dose, $220 \mathrm{mg}$ in $125 \mathrm{ml}$ ) and $5.28 \mathrm{mg} / \mathrm{ml}$ (high-dose, $660 \mathrm{mg}$ in $125 \mathrm{ml}$ ) TFV in iso-osmolar solution. For this study, we collected colorectal biopsy samples from four different research participants at two different time points and concentrations. Therefore, we analyzed 16 biopsy samples using MALDI MSI. In addition to biopsies collected after topical TFV treatments, colorectal biopsies were also obtained from two MSM research participants who were taking TFV orally as TDF (300 mg) for PrEP under a separate JHMIRB-approved clinical protocol after written informed consent.

Preparation of Tissue Samples for MALDI Mass Spectrometry Imaging and Matrix Application. Colorectal tissue samples were individually embedded in OCT (embedding medium for frozen tissue specimens to ensure optimal cutting temperature; Sakura Finetek, Inc., Torrance, CA) and were snap-frozen using dry ice/acetone cooling bath mixture. Tissue sections (20 $\mu \mathrm{m}$ thickness) were next prepared at $-20^{\circ} \mathrm{C}$ using a Leica CM3050S cryostat (Leica Biosystems, Buffalo Grove, IL). The tissue sections were individually mounted onto glass microscope slides (Fisherbrand Superfrost Plus). Matrix [CHCA, $10 \mathrm{mg} / \mathrm{ml}$ in $\left.\mathrm{ACN}: \mathrm{H}_{2} \mathrm{O}(50: 50, \mathrm{v} / \mathrm{v})\right]$ was then applied to the tissue sections using a TM-Sprayer (HTX Technologies, LLC, Chapel Hill, NC) at a flow rate of $100 \mu \mathrm{l} / \mathrm{min}$. The TM-Sprayer was operated at an air pressure $\left(\mathrm{N}_{2}\right)$ of $10 \mathrm{psi}$, spray nozzle velocity of $1200 \mathrm{~mm} / \mathrm{min}$, track spacing at $2 \mathrm{~mm}$, and spray nozzle temperature at $80^{\circ} \mathrm{C}$. Sixteen passes (matrix deposition cycles) were employed for homogeneous deposition of matrix onto colorectal tissue sections.

Tissue extinction coefficient (TEC) values were calculated by dividing the relative ion abundance of TFV (or TFV-DP) in tissue (BioreclamationIVT, Baltimore, MD) by the relative ion abundance of TFV (or TFV-DP) when spotted directly onto a slide using a mixture of TFV and TFV-DP $(50 \mu \mathrm{M})$ as previously described (Bonnel et al., 2018).

MALDI Mass Spectrometry Imaging. MALDI MSI experiments were performed using a LTQ Orbitrap XL (Thermo Fisher Scientific, Bremen, Germany), equipped with a Fourier transform mass spectrometer (FTMS) with the MALDI ion source fitted with a direct beam $\mathrm{N}_{2}$ laser $(\lambda=337.7 \mathrm{~nm})$. Data acquisition was performed using the positive ion mode of the MS instrument at a mass range of $m / z 100-1000$ Da. Mass resolution of the FTMS analyzer was 60,000. CHCA matrix was used at $10 \mathrm{mg} / \mathrm{ml}$ for ionization of both TFV and TFV-DP. A laser energy of $7.8 \mu \mathrm{J}$ was employed for ionization of TFV and TFV-DP. The same MALDI MS conditions including CHCA matrix were used to generate the distribution profiles of three lipid species; phosphatidyl choline (PC) (34:1), PC (16:0/OH), and PC (36:2).

Tissue sections of colorectal biopsy samples were used for tissue imaging experiments. To define the regions of interest, MALDI position files were created by scanning the tissue sample plates with the MALDI camera. Spatial resolution of $50 \mu \mathrm{m}$ was used to create ion images. Mass spectrometry data processing and analysis were done using Xcalibur 3.0 (Thermo Fisher Scientific) and ion images were generated using ImageQuest 1.0.1 software (Thermo Fisher Scientific, San Jose, CA).

Histochemical Analyses. Standard histological techniques were used at the Oncology Tissue Services Laboratory, Johns Hopkins University School of Medicine. Hematoxylin and eosin (H\&E) stain was performed on serial tissue sections (10 $\mu \mathrm{m}$ thick) to visualize the 

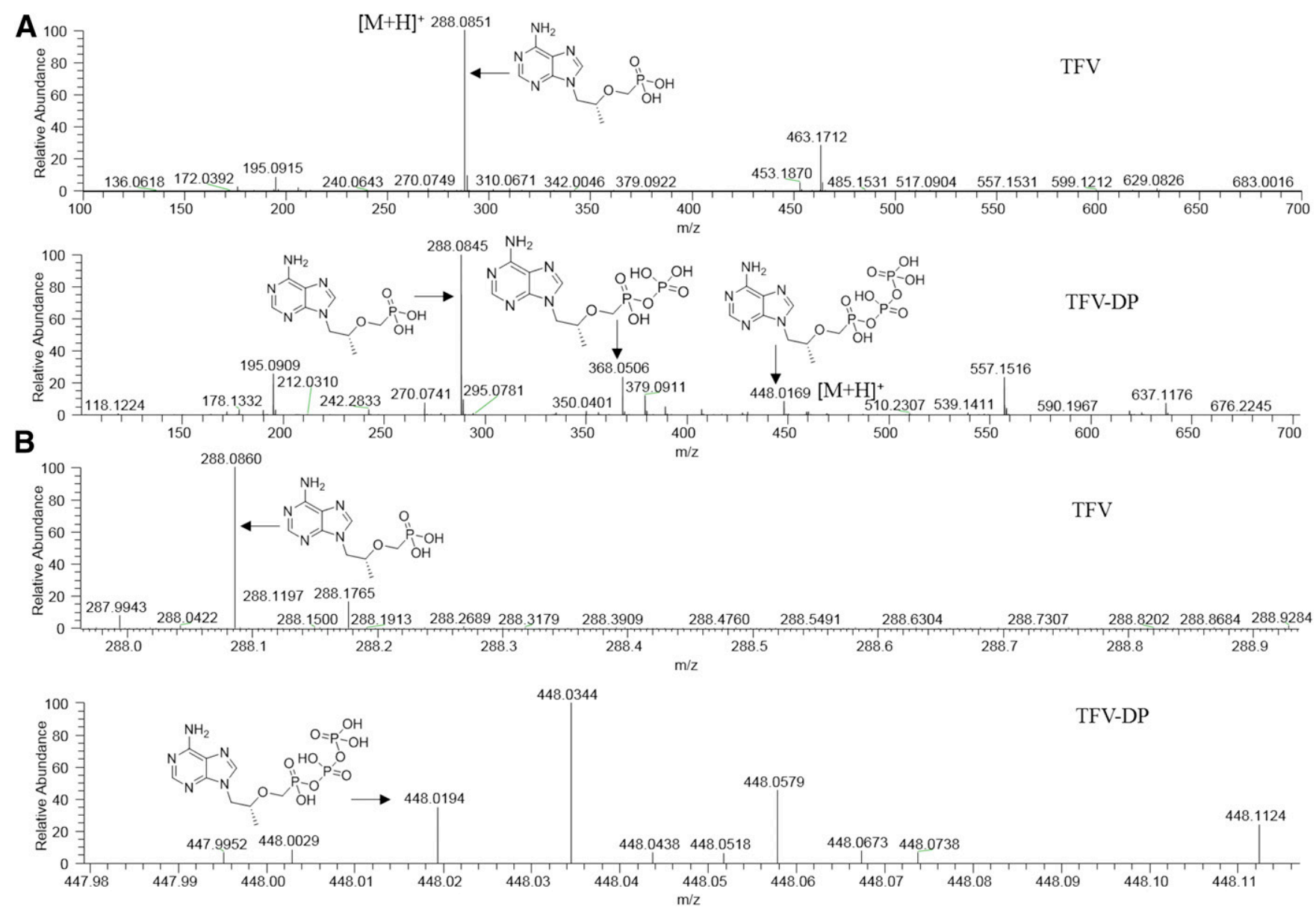

Fig. 1. MALDI full scan mass spectra of TFV and TFV-DP. MALDI full scan mass spectra showing ionization of (A, upper panel) TFV and (A, lower panel) TFV-DP standards in the positive ion mode of the instrument. Representative average full scan mass spectra of (B, upper panel) TFV and (B, lower panel) TFV-DP in colorectal tissue exhibiting the detection of their molecular ions at $\mathrm{m} / z 288.0860$ and 448.0194, respectively, in the positive ion mode of MALDI MS instrument. The matrix used was CHCA.

tissue anatomy and orientation. Immunohistochemistry studies were done using antibodies for CD4 and CD11c cells to localize T cells and dendritic cells, respectively.

\section{Results}

MALDI MS Analysis of TFV and TFV-DP Standards. The primary criterion for matrix selection was the ionization efficiency of TFV and TFV-DP. Therefore, to facilitate detection of TFV and TFV-DP, initially, four commonly used matrices, CHCA, 2, 5-dihydroxybenzoic acid, 3-hydroxypicolinic acid, and 9-aminoacridine were tested. From these initial experiments, TFV and TFV-DP exhibited the greatest ion intensities for their molecular ions at $\mathrm{m} / z 288.0851$ (1.7 ppm) and 448.0169 (3.1 ppm), respectively, using the CHCA matrix and positive ion mode (Fig. 1A). Additionally, fragment ions resulting from the loss of phosphate groups at $\mathrm{m} / \mathrm{z} 368.0506$ and 288.0845 corresponding to $\mathrm{C}_{9} \mathrm{H}_{16} \mathrm{~N}_{5} \mathrm{O}_{7} \mathrm{P}_{2}^{+}$and $\mathrm{C}_{9} \mathrm{H}_{15} \mathrm{~N}_{5} \mathrm{O}_{4} \mathrm{P}^{+}$, respectively, were detected for TFV-DP (Fig. 1A, lower panel). TFV was detectable as its $[\mathrm{M}+\mathrm{H}]^{+}$at $\mathrm{m} / z 288.0849$ using 2, 5-dihydroxybenzoic acid as the matrix; however, the ion abundance was low (data not shown). TFV-DP was not readily detected using 2, 5-dihydroxybenzoic acid, 3-hydroxypicolinic acid, and 9 -aminoacridine as matrices. TEC values for TFV and
TFV-DP at $50 \mu \mathrm{M}$ were 0.014 and 0.006 , respectively. The prediction of $\log P$ values were performed using ALOGPS 2.1 software (Tetko et al., 2005). The predicted $\log P$ value of TFV was -1.74 whereas TFV-DP had a predicted $\log P$ value of -2.75 .

MALDI MSI of TFV and TFV-DP in Colorectal Tissue Obtained from Subjects Who Received TFVContaining Enemas. To investigate the spatial distribution of both TFV and TFV-DP in human colorectal tissue following topical application, MALDI MSI experiments were performed using biopsy samples obtained from healthy volunteers who received $125-\mathrm{ml}$ enemas containing either $220(1.76 \mathrm{mg} / \mathrm{ml})$ or $660 \mathrm{mg}(5.78 \mathrm{mg} / \mathrm{ml})$ of TFV. In these samples, we detected both TFV and TFV-DP as their corresponding molecular ions at $m / z 288.0860(1.3 \mathrm{ppm})$ and $448.0194(2.4 \mathrm{ppm})$, respectively (Fig. 1B). Tissue anatomy and orientation were visualized using H\&E stain (Fig. 2A, upper panel). MALDI ion images revealed the heterogeneous distribution of both TFV and TFV-DP across the colorectal tissue section (Fig. 2, B and $\mathrm{C}$, upper panel). To further confirm the specificity of heterogeneous distribution of TFV and TFV-DP distribution, we simultaneously generated ion images for three different lipid species, PC (34:1), PC (16:0/OH), and PC (36:2). Interestingly, the ion images corresponding to the molecular ions of the above three different lipid species at $m / z 760.5856$ 

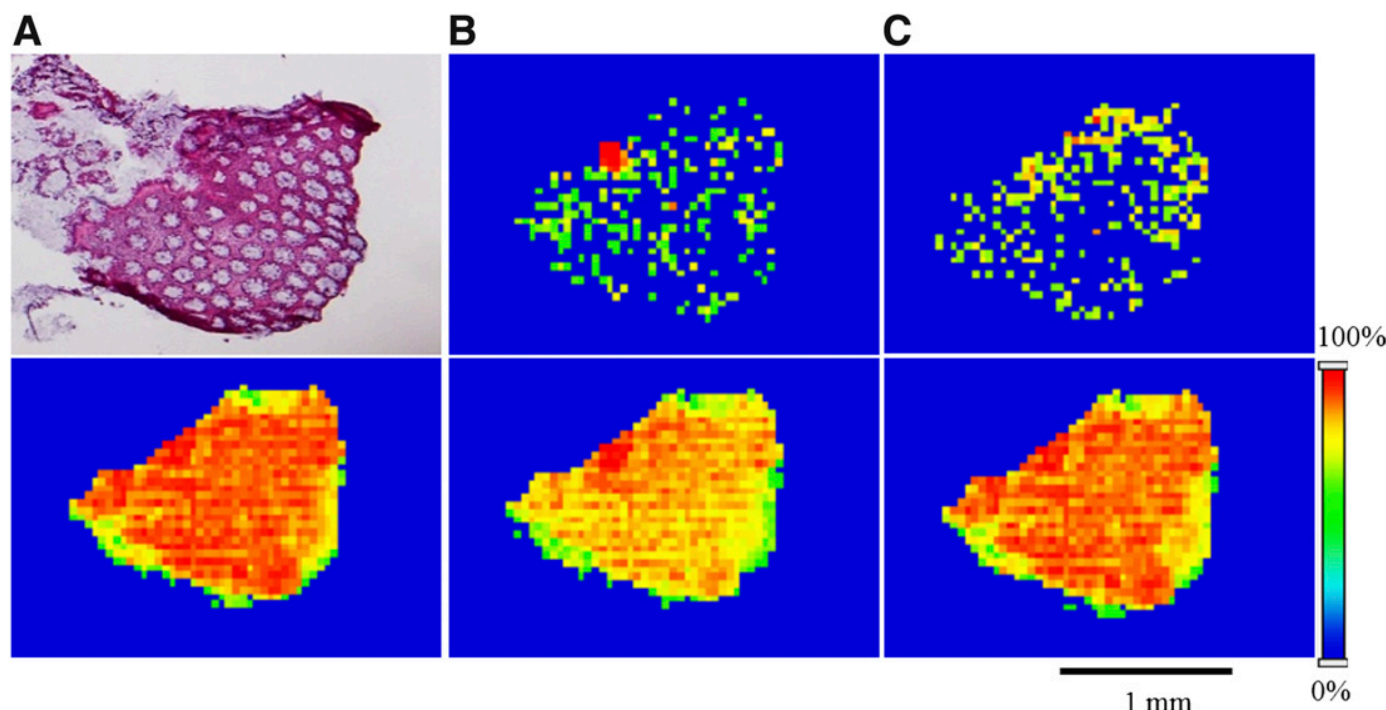

Fig. 2. Spatial distribution of TFV, TFV-DP, PC (34:1), PC (16:0/OH), and PC (36:2) in a colorectal tissue section of a subject received a TFV-containing enema. (A, upper panel) H\&E stain indicating tissue anatomy and orientation. MALDI MS ion images of (B, upper panel) TFV and (C, upper panel) TFV-DP revealing their heterogeneous distribution across the colorectal tissue section. In contrast, lipid species, (A, lower panel) PC (34:1), (B, lower panel) PC (16:0/OH), and (C, lower panel) PC (36:2) exhibiting relative homogeneity in their distribution. Spatial resolution for MALDI MS ion images was $50 \mu \mathrm{m}$. The red color represents the highest signal intensity (100\%), whereas the blue color represents the lowest signal (0\%) of the ion of interest. Scale bar, $1 \mathrm{~mm}$.

(0.0 ppm), 496.3403 (0.0 ppm), and $786.6013(0.1 \mathrm{ppm})$ showed relative homogeneous distribution across the tissue section (Fig. 2, lower panel).

Coupling MALDI MSI Analyses with Immunohistochemistry. CD4 T cells are the most relevant cell type for HIV infection since they are the primary sites of HIV infection. Additionally, dendritic cells play multiple roles at different stages of HIV infection, thereby representing a vital subset in HIV infection in addition to CD4 T cells (Manches et al., 2014). Therefore, as the next step, we looked at the distribution of CD4 $\mathrm{T}$ cells and CD11c dendritic cells to determine whether a correlation between TFV and/or TFV-DP spatial distribution and the localization of CD4 and CD11c cells could be observed in colorectal tissue. On the basis of immunohistochemical analyses, regions that had CD4 and CD11c cells exhibited a brown color in tissue sections (Fig. 3). However, from these data, we were not able to see any direct correlation between TFV and/or TFV-DP spatial distribution and the localization of CD4 cells and CD11c cells in colorectal tissue (Fig. 3).
Dose and Time Dependence of TFV and TFV-DP Distribution. To study the dose dependence of TFV and TFV-DP distribution in human colorectal tissue following topical application, two different enema doses of TFV, i.e., $1.76 \mathrm{mg} / \mathrm{ml}$ (low-dose) and $5.28 \mathrm{mg} / \mathrm{ml}$ (high-dose) were analyzed. For time-dependence studies, colorectal biopsies were collected at 3 and 24 hours after enema administration. From these, both TFV and TFV-DP were detected in colorectal biopsies of three research participants, J001, J003, and J004, collected 3 hours following low-dose enema treatment, whereas ion abundances for both TFV and TFV-DP were relatively low in the colorectal tissue of research participant J002 that was analyzed (Fig. 4A). Moreover, after 3-hour highdose TFV enema treatment, only two research participants, J001 and J004, exhibited detectable TFV and TFV-DP levels in their colorectal biopsies (Fig. 4B). Corresponding ion maps of TFV and TFV-DP showed their heterogeneous distribution across the colorectal tissue (Fig. 4, A and B). On the other hand, both TFV and TFV-DP were not detected at relatively high ion abundances in colorectal biopsy samples of all
CD4

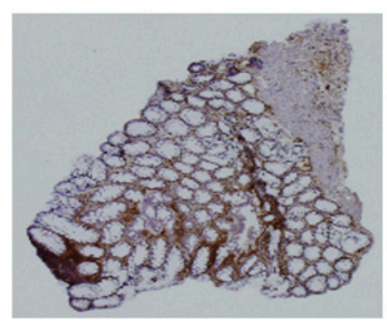

CD11c

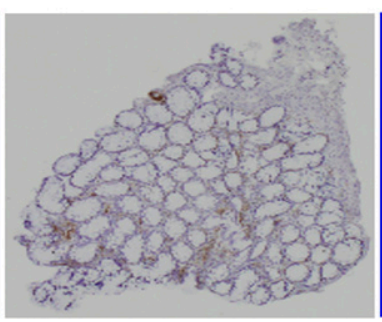

TFV

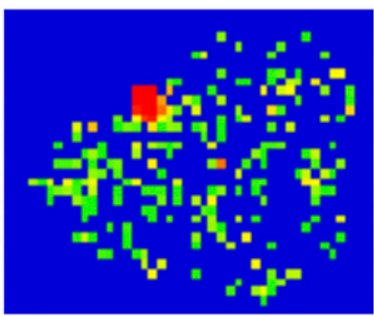

TFV-DP

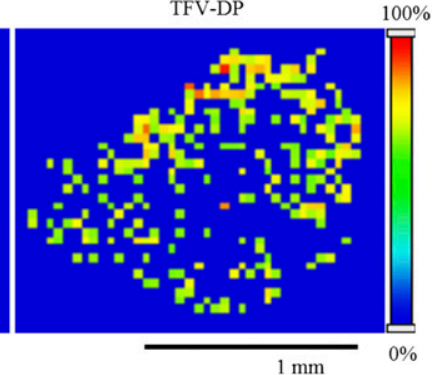

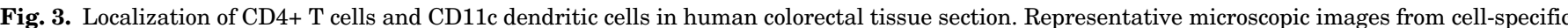

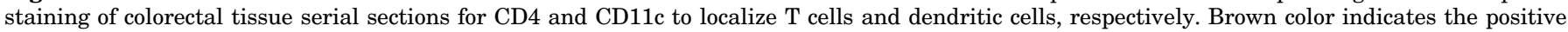

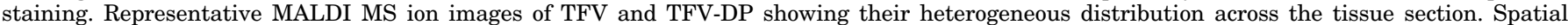
resolution for MALDI MS images, $50 \mu \mathrm{m}$. Scale bar, $1 \mathrm{~mm}$. 

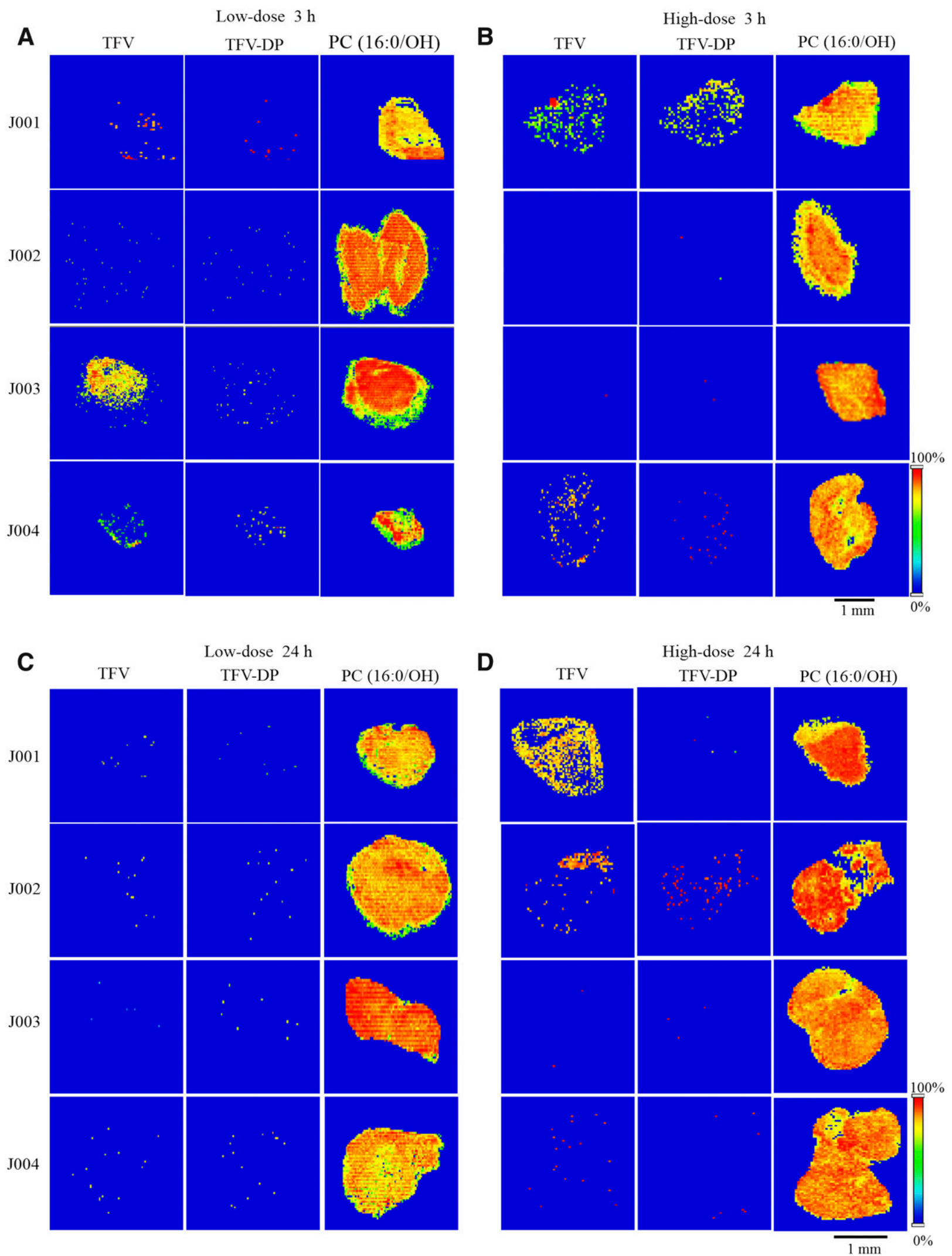

Fig. 4. Time and dose dependence of TFV and TFV-DP distribution in human colorectal tissue. Representative MALDI MS ion images of (left) TFV, (middle) TFV-DP, and (right) PC(16:0/OH) in colorectal biopsy sections of four different research participants, J001, J002, J003, and J004 collected (A) 3 hours following low-dose, (B) 3 hours following high-dose, (C) 24 hours following low-dose, and (D) 24 hours following high-dose TFV- containing enema treatment. Spatial resolution for MALDI MS images, $50 \mu \mathrm{m}$. Scale bar, $1 \mathrm{~mm}$. 
subjects collected 24 hours following low-dose enema administration (Fig. 4C). By contrast, corresponding TFV ion images revealed its heterogeneous localization in biopsies of two research participants, J001 and J002, at high-dose treatment (Fig. 4D). Nevertheless, TFV-DP was detected at relatively high abundance only in the biopsy sample of J002 at 24 hours following high-dose enema treatment (Fig. 4D). It should be noted that one of the lipid species we imaged simultaneously, PC (16:0/OH), was detected in all the biopsies examined and found to be relative homogeneous in its distribution across the colorectal tissue (Fig. 4).

Next, we wanted to test whether these findings could be extended to oral dosing. To do this, we obtained colorectal biopsies from two research participants taking TDF for PrEP. As previously observed for the biopsies obtained following topical TFV application, both TFV and TFV-DP exhibited heterogeneity in their spatial distribution (Fig. 5, B, C, E, and $\mathrm{F}$, upper panel) including the presence of regions where TFV and TFV-DP were not detectable. Commensurate with our previously mentioned analyses, the spatial distribution of PC (34:1), PC (16:0/OH), and PC (36:2) was relatively homogeneous across the colorectal tissue (Fig. 5, lower panel).

\section{Discussion}

We demonstrated the heterogeneous colorectal tissue distribution of TFV and TFV-DP following a rectal dose of TFV as enema and after an oral dose of TDF. This included the finding that regions of tissue exist in which TFV and TFV-DP are not detectable. These observations have not been previously reported and were only facilitated by our development and application of a MALDI MSI approach. Of note, although it has been reported that TFV and TFV-DP can be detected in homogenates of colorectal tissue even after 14 days by using liquid chromatography tandem mass spectrometry (Hawkins et al., 2005; Patterson et al., 2011), our data raise the possibility that TFV and TFV-DP may not be detectable throughout the tissue. The spatial profiles that we generated for TFV and TFV-DP did not show complete overlap and TFV-DP appeared to be less abundant than TFV, despite the fact that the limits of detection were the same for both analytes and TFV-DP has a longer half-life than TFV (Hawkins et al., 2005; Patterson et al., 2011; Louissaint et al., 2013). Further, our data suggest differential distribution of TFV and TFV-DP among study subjects. Of note, inter-subject variability of TFV and TFV-DP concentrations has been previously reported following fixed oral doses of TFV using liquid chromatography tandem mass spectrometry-based detection (Hendrix et al., 2016). Moreover, in the same study, inter-subject coefficients of variation were determined to range from $33 \%$ to $82 \%, 50 \%$ to $121 \%$, and $46 \%$ to $71 \%$, respectively, for serum TFV, TFV-DP in peripheral blood mononuclear cells, and TFV-DP in CD4 T cells (Hendrix et al., 2016). Particularly relevant to the present study, inter-subject variability in TFV and TFV-DP concentrations in cells and colorectal tissue have been reported following both oral and topical TFV dosing. In tissue homogenates, coefficients of variation for TFV-DP ranged from $0 \%$ to $307 \%, 85 \%$ to $312 \%$, and $132 \%$ for single oral, single rectal, and multiple rectal dosing, respectively (Yang et al., 2014). Likewise, for single oral, single rectal, and multiple rectal dosing, TFV coefficients of variation were $112 \%-364 \%, 0 \%-220 \%$ and
$236 \%$, respectively (Yang et al., 2014). Our data add to these findings by demonstrating that inter-individual variability exists not only in overall concentrations of TFV and TFV-DP in tissue, but in the distribution of these compounds within a biopsy as well.

The physicochemical properties of TFV and TFV-DP, such as molecular weight, lipophilicity, protein binding, and interactions with endogenous cellular compounds, may contribute to the differences in spatial distribution in colorectal tissue. Interestingly, from our analyses, TFV-DP exhibited a lower $\log P$ value, -2.75 compared with TFV, -1.74 . This observation indicates that TFV-DP may have been less readily detectable in the colorectal tissue biopsies owing to the ability of TFV-DP to form stronger interactions with endogenous cellular components than does TFV. Of note, it has been reported that there is a correlation between the MALDI MSI sensitivity and analyte lipophilicity (Log $P$ value) (Bonnel et al., 2018). Therefore, the observed relationship between Log $P$ values of TFV and TFV-DP and their differential detection in tissue reveals a potential factor that could contribute to the observed TFV and TFV-DP distributions in tissue. To further consider the possibility that differential binding of TFV and TFV-DP to components of tissue contribute to the spatial distributions that we observed for these molecules, we estimated the tissue binding potential by determining the TEC. TFV had a TEC value of 0.014 that was slightly higher than that of TFV-DP, 0.006. That is, TFV exhibited greater ionization than TFV-DP. Taken together, these data suggest that both the Log $\mathrm{P}$ and TEC values of TFV-DP could provide explanations for why TFV-DP was not detected in certain regions of tissue. Of importance, by developing our MALDI MSI approach to simultaneously detect three different lipid species, PC (34:1), PC (16:0/OH), and PC (36:2), in addition to TFV and TFV-DP, we were able to validate our MALDI MSI method and rule out the possibility that processing of the biopsies was responsible for the heterogeneity in TFV and TFV-DP distribution that we found. This was attributable to the fact that the lipids we monitored were relatively homogeneously distributed in the colorectal tissue biopsies and these data were concordant with previous studies on lipid distributions (van Meer et al., 2008).

Heterogeneity of TFV-DP among cell types has been reported previously, as concentrations of TFV-DP in CD4 cells differ from those in the total colorectal tissue homogenates and total cell populations (Yang et al., 2014). CD4 cells are known to be the sites of HIV infection and hence the most relevant cell type for HIV infection (Masur et al., 1989; Okoye and Picker, 2013). In addition, CD11c dendritic cells are also of interest as one of the first cell types to experience HIV infection and they are also widely distributed in mucosal tissue (Manches et al., 2014). We identified regions of CD4 and CD11c cells in colorectal tissue where TFV-DP was present; however, there were also regions in which these cells were abundant but TFV-DP was undetectable. Thus, our data suggest that CD4 and CD11c cells do not drive TFV and/or TFV-DP spatial distribution in colorectal tissue.

In addition, TFV is known to be a substrate for efflux transporter multidrug resistance protein type 4 (MRP4), a member of ABC family, from both in vitro and clinical studies (Ray et al., 2006; Imaoka et al., 2007). In another study, Mallants et al. (2005) demonstrated TFV as a substrate of MRP2; however, its role in intracellular TFV disposition was 


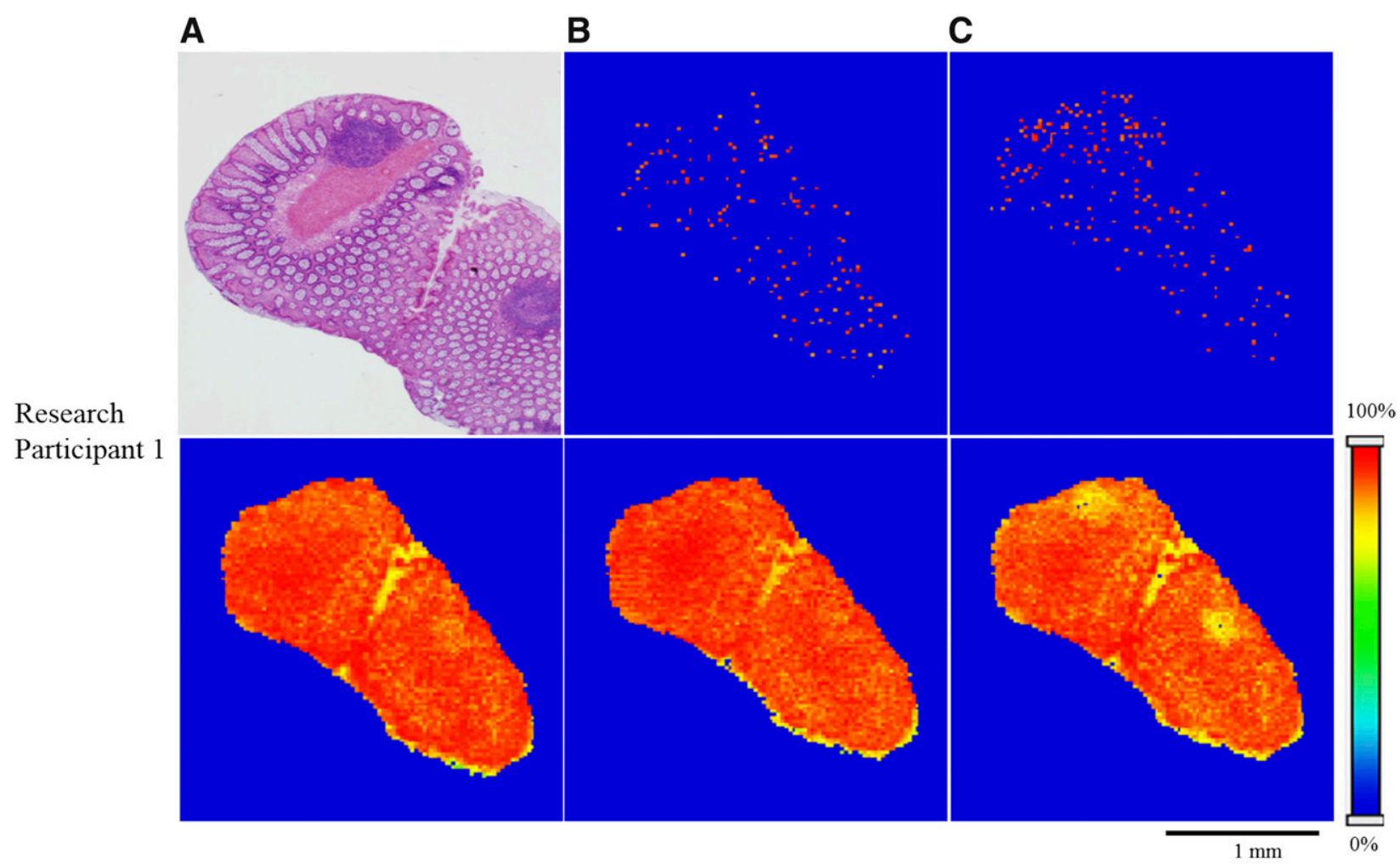

D

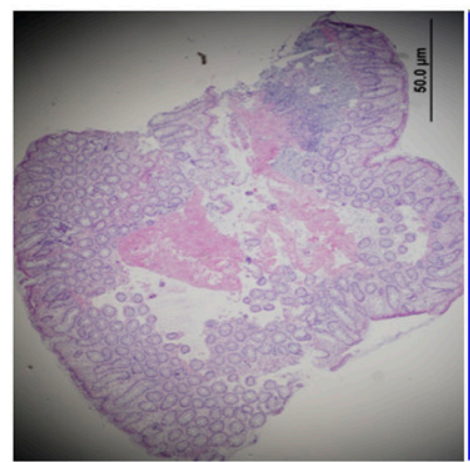

Research

Participant 2

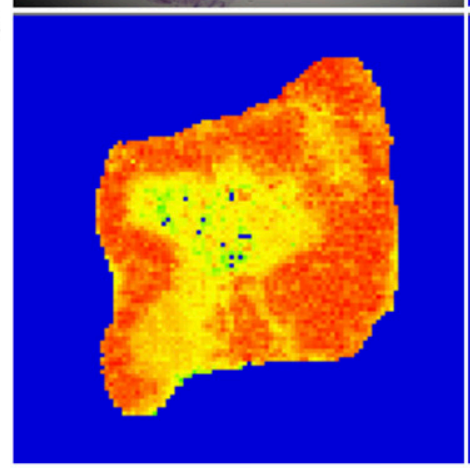

$\mathbf{E}$
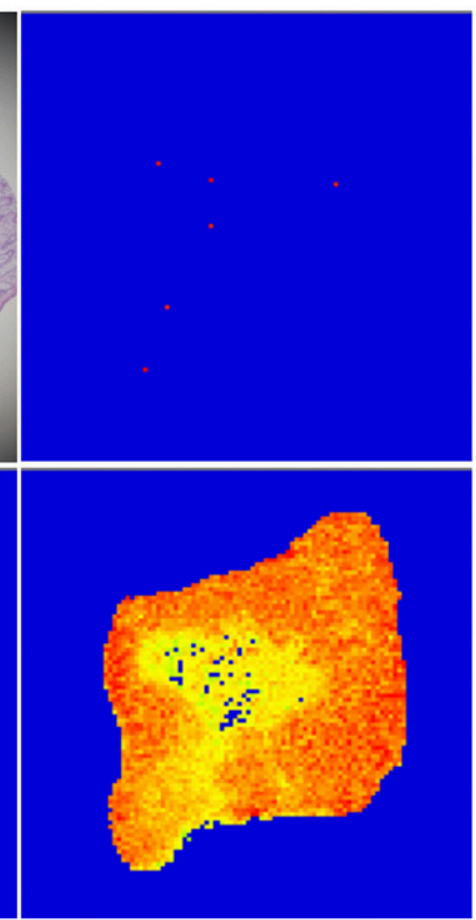

$\mathbf{F}$
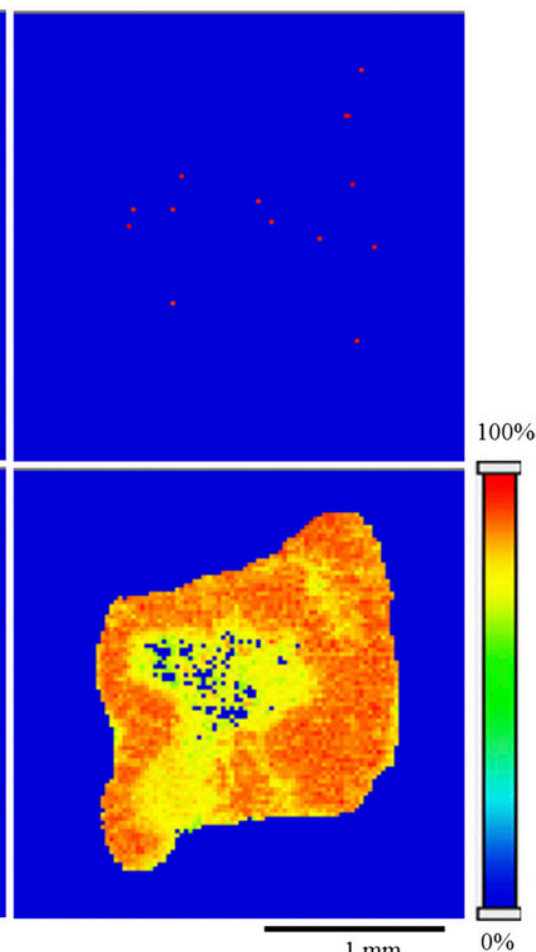

Fig. 5. Spatial distribution of TFV and TFV-DP in colorectal tissue sections of two research participants received oral TFV as TFV disoproxil fumarate. (A and D, upper panel) H\&E stain exhibiting tissue anatomy and orientation. Representative MALDI MS ion images of (B and E, upper panel) TFV and (C and F, upper panel) TFV-DP revealing the heterogeneity in their distribution. In contrast, lipid species, (A and D, lower panel) PC (34:1), (B and E, lower panel) PC (16:0/OH), and (C and F, lower panel) PC (36:2) showing relative homogeneity in their distribution. Spatial resolution for MALDI MS ion images was $50 \mu \mathrm{m}$. Red represents the highest signal intensity (100\%), blue represents the lowest signal (0\%) of the ion of interest. Scale bar, $1 \mathrm{~mm}$. 
unclear. Beyond the efflux transporters, TFV has also been implicated as a substrate for solute carrier family transporters that facilitate drug uptake into cells; human organic anion transporter (OAT1) and OAT3 (Bleasby et al., 2005; Kiser et al., 2008). Further, in considering the distribution of TFV-DP in colorectal tissue, the local expression and activity of both kinases that catalyze the phosphorylation of TFV and phosphatases that dephosphorylate TFV-DP represent additional variables.

To take our work further, we also ascertained the localization of TFV and TFV-DP in colorectal tissue of two subjects who received oral TFV as TDF. Through this, we found that, as with the spatial distributions in colorectal tissue of TFV and TFV-DP observed following topical dosing, TFV and TFV-DP were heterogeneously distributed in colorectal tissue of subjects receiving oral TDF. However, the relative ion abundances of both TFV and TFV-DP in the biopsies from orally dosed subjects were low compared with topical application. This observation is likely due to the differences in dosing route as previously described (Yang et al., 2014; Taneva et al., 2015). Previous studies by Anton et al. (2012) and Hendrix et al. (2013) have demonstrated higher accumulation of TFV-DP, i.e., 6- to 10-fold increase in colorectal tissue homogenate following rectal dosing (40 mg TFV per dose), compared with oral dosing (300 mg TDF).

In this study, we determined the spatial distribution of TFV and TFV-DP in human colorectal tissue using a state-ofthe-art technique, MALDI MSI. This work provides an important proof-of-concept; however, to further enhance understanding of TFV/TFV-DP in colorectal tissue, future studies using larger sample sizes are required. Although we performed qualitative analysis on TFV and TFV-DP spatial distribution in the present study, to take these findings further it will be important for future work to focus on quantitative determinations of TFV and TFV-DP concentrations across tissue. Further, future work is required to determine the factors that drive the heterogeneity of the spatial distribution of TFV and TFV-DP in colorectal tissue. Nevertheless, our data obtained through this approach provide the first visualization of TFV and TFV-DP in a mucosal tissue that is a putative site of HIV infection. Importantly, we demonstrate that there are regions of tissue that are devoid of detectable TFV and/or TFV-DP. These data can be leveraged in optimizing TFV dosing and formulations to identify those that provide increased exposure to TFV and TFV-DP in tissue overall. Taken together, the results from this study provide new insights into the disposition of topical TFV in colorectal tissue.

\section{Authorship Contributions}

Participated in research design: Seneviratne, Hendrix, Fuchs, Bumpus.

Conducted experiments: Seneviratne.

Performed data analysis: Seneviratne, Hendrix, Bumpus.

Wrote or contributed to the writing of the manuscript: Seneviratne, Hendrix, Fuchs, Bumpus.

\section{References}

Abdool Karim Q, Abdool Karim SS, Frohlich JA, Grobler AC, Baxter C, Mansoor LE, Kharsany AB, Sibeko S, Mlisana KP, Omar Z, et al.; CAPRISA 004 Trial Group (2010) Effectiveness and safety of tenofovir gel, an antiretroviral microbicide, for the prevention of HIV infection in women. Science 329:1168-1174.

Angel PM and Caprioli RM (2013) Matrix-assisted laser desorption ionization imaging mass spectrometry: in situ molecular mapping. Biochemistry 52:3818-3828.
Anton PA, Cranston RD, Kashuba A, Hendrix CW, Bumpus NN, Richardson-Harman N, Elliott J, Janocko L, Khanukhova E, Dennis R, et al. (2012) RMP-02/MTN-006: a phase 1 rectal safety, acceptability, pharmacokinetic, and pharmacodynamic study of tenofovir 1\% gel compared with oral tenofovir disoproxil fumarate. AIDS Res Hum Retroviruses 28:1412-1421.

Baeten J and Celum C (2013) Systemic and topical drugs for the prevention of HIV infection: antiretroviral pre-exposure prophylaxis. Annu Rev Med 64: 219-232.

Bauermeister JA, Meanley S, Pingel E, Soler JH, and Harper GW (2013) PrEP awareness and perceived barriers among single young men who have sex with men. Curr HIV Res 11:520-527.

Bleasby K, Hall LA, Perry JL, Mohrenweiser HW, and Pritchard JB (2005) Functional consequences of single nucleotide polymorphisms in the human organic anion transporter hOAT1 (SLC22A6). J Pharmacol Exp Ther 314:923-931.

Bonnel D, Legouffe R, Eriksson AH, Mortensen RW, Pamelard F, Stauber J, and Nielsen KT (2018) MALDI imaging facilitates new topical drug development process by determining quantitative skin distribution profiles. Anal Bioanal Chem 410:2815-2828.

Carballo-Diéguez A, Balán IC, Brown W, III, Giguere R, Dolezal C, Leu CS, Marzinke MA, Hendrix CW, Piper JM, Richardson BA, et al. (2017) High levels of adherence to a rectal microbicide gel and to oral pre-exposure prophylaxis ( $\mathrm{PrEP})$ achieved in MTN-017 among men who have sex with men (MSM) and transgender women. PLoS One 12: 0181607.

De Clercq E (2007) Acyclic nucleoside phosphonates: past, present and future. Bridging chemistry to HIV, HBV, HCV, HPV, adeno-, herpes-, and poxvirus infections: the phosphonate bridge. Biochem Pharmacol 73:911-922.

Dolezal C, Frasca T, Giguere R, Ibitoye M, Cranston RD, Febo I, Mayer KH, McGowan I, and Carballo-Diéguez A (2015) Awareness of post-exposure prophylaxis (PEP) and pre-exposure prophylaxis (PrEP) is low but interest is high among men engaging in condomless anal sex with men in Boston, Pittsburgh, and San Juan. AIDS Educ Prev 27:289-297.

Eaton LA, Driffin DD, Bauermeister J, Smith H, and Conway-Washington C (2015) Minimal awareness and stalled uptake of pre-exposure prophylaxis (PrEP) among at risk, HIV-negative, black men who have sex with men. AIDS Patient Care STDS 29:423-429.

Greene GJ, Swann G, Fought AJ, Carballo-Diéguez A, Hope TJ, Kiser PF, Mustanski B, and D'Aquila RT (2017) Preferences for long-acting pre-exposure prophylaxis (PrEP), daily oral PrEP, or condoms for HIV prevention among U.S. men who have sex with men. AIDS Behav 21:1336-1349.

Greer T, Sturm R, and Li L (2011) Mass spectrometry imaging for drugs and metabolites. J Proteomics 74:2617-2631.

Hawkins T, Veikley W, St Claire RL, III, Guyer B, Clark N, and Kearney BP (2005) Intracellular pharmacokinetics of tenofovir diphosphate, carbovir triphosphate, and lamivudine triphosphate in patients receiving triple-nucleoside regimens. $J$ Acquir Immune Defic Syndr 39:406-411.

Hendrix CW, Andrade A, Bumpus NN, Kashuba AD, Marzinke MA, Moore A, Anderson PL, Bushman LR, Fuchs EJ, Wiggins I, et al. (2016) Dose frequency ranging pharmacokinetic study of tenofovir-emtricitabine after directly observed dosing in healthy volunteers to establish adherence benchmarks (HPTN 066). AIDS Res Hum Retroviruses 32:32-43.

Hendrix CW, Chen BA, Guddera V, Hoesley C, Justman J, Nakabiito C, Salata R Soto-Torres L, Patterson K, Minnis AM, et al. (2013) MTN-001: randomized pharmacokinetic cross-over study comparing tenofovir vaginal gel and oral tablets in vaginal tissue and other compartments. PLoS One 8:e55013.

Imaoka T, Kusuhara H, Adachi M, Schuetz JD, Takeuchi K, and Sugiyama Y (2007) Functional involvement of multidrug resistance-associated protein 4 (MRP4/ABCC4) in the renal elimination of the antiviral drugs adefovir and tenofovir. Mol Pharmacol 71: 619-627.

Kearney BP, Flaherty JF, and Shah J (2004) Tenofovir disoproxil fumarate: clinical pharmacology and pharmacokinetics. Clin Pharmacokinet 43:595-612.

Kiser JJ, Aquilante CL, Anderson PL, King TM, Carten ML, and Fletcher CV (2008) Clinical and genetic determinants of intracellular tenofovir diphosphate concentrations in HIV-infected patients. J Acquir Immune Defic Syndr 47:298-303.

Lade JM, To EE, Hendrix CW, and Bumpus NN (2015) Discovery of genetic variants of the kinases that activate tenofovir in a compartment-specific manner. EBioMedicine 2:1145-1152.

Louissaint NA, Cao YJ, Skipper PL, Liberman RG, Tannenbaum SR, Nimmagadda S, Anderson JR, Everts S, Bakshi R, Fuchs EJ, et al. (2013) Single dose pharmacokinetics of oral tenofovir in plasma, peripheral blood mononuclear cells, colonic tissue, and vaginal tissue. AIDS Res Hum Retroviruses 29:1443-1450.

Mallants R, Van Oosterwyck K, Van Vaeck L, Mols R, De Clercq E, and Augustijns P (2005) Multidrug resistance-associated protein 2 (MRP2) affects hepatobiliary elimination but not the intestinal disposition of tenofovir disoproxil fumarate and its metabolites. Xenobiotica 35:1055-1066.

Manches O, Frleta D, and Bhardwaj N (2014) Dendritic cells in progression and pathology of HIV infection. Trends Immunol 35:114-122.

Masur H, Ognibene FP, Yarchoan R, Shelhamer JH, Baird BF, Travis W, Suffredini AF, Deyton L, Kovacs JA, Falloon J, et al. (1989) CD4 counts as predictors of opportunistic pneumonias in human immunodeficiency virus (HIV) infection. Ann Intern Med 111:223-231.

Miller MD, Margot NA, Hertogs K, Larder B, and Miller V (2001) Antiviral activity of tenofovir (PMPA) against nucleoside-resistant clinical HIV samples. Nucleosides Nucleotides Nucleic Acids 20:1025-1028.

Mitchell JW, Lee JY, Woodyatt C, Bauermeister J, Sullivan P, and Stephenson R (2016) HIV-negative male couples' attitudes about pre-exposure prophylaxis (PrEP) and using PrEP with a sexual agreement. AIDS Care 28:994-999.

Naesens L, Bischofberger N, Augustijns P, Annaert P, Van den Mooter G, Arimilli MN, Kim CU, and De Clercq E (1998) Antiretroviral efficacy and pharmacokinetics of oral bis(isopropyloxycarbonyloxymethyl)-9-(2-phosphonylmethoxypropyl)adenine in mice. Antimicrob Agents Chemother 42:1568-1573. 
Okoye AA and Picker LJ (2013) CD4(+) T-cell depletion in HIV infection: mechanisms of immunological failure. Immunol Rev 254:54-64.

Patterson KB, Prince HA, Kraft E, Jenkins AJ, Shaheen NJ, Rooney JF, Cohen MS, and Kashuba AD (2011) Penetration of tenofovir and emtricitabine in mucosal tissues: implications for prevention of HIV-1 transmission. Sci Transl Med 3:112re4.

Ray AS, Cihlar T, Robinson KL, Tong L, Vela JE, Fuller MD, Wieman LM, Eisenberg EJ, and Rhodes GR (2006) Mechanism of active renal tubular efflux of tenofovir. Antimicrob Agents Chemother 50:3297-3304.

Robbins BL, Srinivas RV, Kim C, Bischofberger N, and Fridland A (1998) Anti-human immunodeficiency virus activity and cellular metabolism of a potential prodrug of the acyclic nucleoside phosphonate 9-R-(2-phosphonomethoxypropyl)adenine (PMPA), Bis(isopropyloxymethylcarbonyl)PMPA. Antimicrob Agents Chemother 42:612-617.

Taneva E, Crooker K, Park SH, Su JT, Ott A, Cheshenko N, Szleifer I, Kiser PF, Frank B, Mesquita PMM, et al (2015) Differential mechanisms of tenofovir and tenofovir disoproxil fumarate cellular transport and implications for topical preexposure prophylaxis. Antimicrob Agents Chemother 60:1667-1675.
Tetko IV, Gasteiger J, Todeschini R, Mauri A, Livingstone D, Ertl P, Palyulin VA, Radchenko EV, Zefirov NS, Makarenko AS, et al. (2005) Virtual computationa chemistry laboratory-design and description. J Comput Aided Mol Des 19 453-463.

van Meer G, Voelker DR, and Feigenson GW (2008) Membrane lipids: where they are and how they behave. Nat Rev Mol Cell Biol 9:112-124.

Yang KH, Hendrix C, Bumpus N, Elliott J, Tanner K, Mauck C, Cranston R, McGowan I, Richardson-Harman N, Anton PA, et al. (2014) A multi-compartment single and multiple dose pharmacokinetic comparison of rectally applied tenofovir $1 \%$ gel and oral tenofovir disoproxil fumarate. PLoS One 9:e106196.

Address correspondence to: Dr. Namandjé N. Bumpus, Department of Medicine, Division of Clinical Pharmacology, The Johns Hopkins University School of Medicine, 725 North Wolfe Street, Biophysics 307, Baltimore, MD 21205. E-mail: nbumpus1@jhmi.edu 